Two - related sample rate test (McNemar test), McNmar Statistics, 18.24, The $\mathrm{P}<0.0001, \mathrm{Kappa}=0.37,95 \% \quad \mathrm{CI}(0.20,0.54)$, The detection rates differ between the two detection methods too.

Conclusions Magnetic resonance has low sensitivity to lymph node transfer determination, high specificity, high positive and negative prediction value, which can be used as a preoperative routine examination, but by magnetic resonance examination alone, to judge whether there is lymph node transfer that is prone to leakage diagnosis, more effective testing methods are needed to assist in the diagnosis.

\section{EPV165/\#137 GYNECOLOGIC CANCER APPOINTMENT ATTENDANCE DURING THE COVID-19 PANDEMIC}

T Ellett*, S Luke, D Schaps, R Previs, H Moss. Duke University School of Medicine, Obstetrics and Gynecology, Durham, USA

\subsection{6/ijgc-2021-IGCS.235}

Objectives The COVID-19 pandemic has significantly disrupted medical care. The purpose of this analysis was to determine the impact of the pandemic on gynecologic cancer appointment adherence.

Methods All appointments scheduled at an academic gynecologic oncology center from March 2019 to January 2021 were included. Appointments were stratified into two groups pre-pandemic (March '19 to January '20) and pandemic (March '20 to January '21). Appointments were determined 'missed' if the patient did not show or cancelled. A multivariable logistic regression was performed to determine the odds ratio (OR) of appointment adherence during the pandemic.

Results 31,803 appointments were scheduled during the study period $(15,834(49.8 \%)$ pre-pandemic and 15,969 $(50.2 \%)$ during the pandemic). There were significantly more appointments missed during the pandemic than prepandemic - $7266(45.5 \%)$ vs. 6131 (38.7\%); p<.0001. The adjusted odds of missing an appointment were significantly higher during the pandemic (OR 1.43 [95\% CI 1.36 to $1.51] ; \mathrm{p}<0.0001)$. There were more return visits missed during the pandemic than before - 6696 (47.0\%) vs 5341 (39.5\%); $\quad \mathrm{p}<0.0001$. New-patient visit adherence was unchanged. Race, ethnicity, and income were not associated with missed appointments.

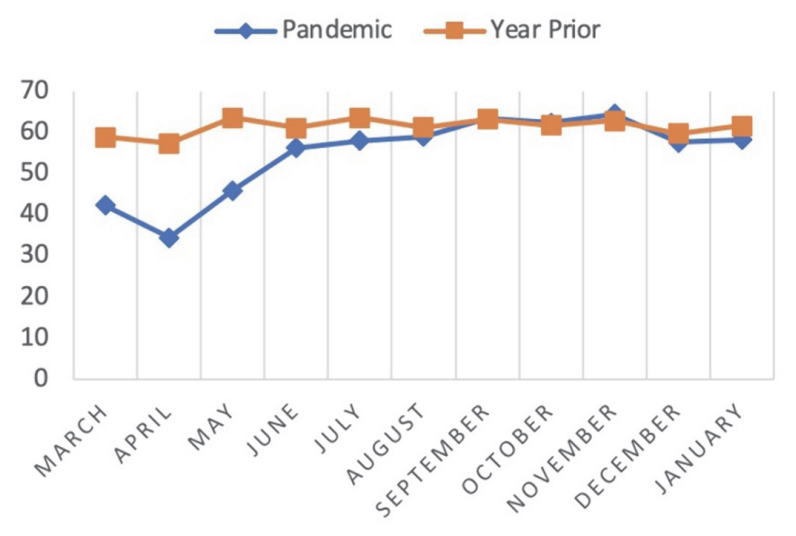

Abstract EPV165/\#137 Figure 1 Line graph of percent-attendance by month stratified by pre-pandemic and during-pandemic
Abstract EPV165/\#137 Table 1 Attendance by month stratified by pre-pandemic and during-pandemic

\begin{tabular}{|l|l|l|l|}
\hline Month & $\begin{array}{l}\text { Year Prior } \\
(\%)\end{array}$ & $\begin{array}{l}\text { Pandemic } \\
(\%)\end{array}$ & p-value \\
\hline March & 58.82 & 42.23 & $<.0001$ \\
\hline April & 57.24 & 34.36 & $<.0001$ \\
\hline May & 63.56 & 45.84 & $<.0001$ \\
\hline June & 61.01 & 56.15 & 0.0074 \\
\hline July & 63.56 & 57.94 & 0.0014 \\
\hline August & 61.09 & 58.97 & 0.2423 \\
\hline September & 63.21 & 63.25 & 0.9827 \\
\hline October & 61.71 & 62.23 & 0.7703 \\
\hline November & 62.72 & 64.38 & 0.3739 \\
\hline December & 59.65 & 57.57 & 0.2601 \\
\hline January & 61.5 & 58.17 & 0.0649 \\
\hline
\end{tabular}

Conclusions There were increased odds of missing an appointment during the pandemic than during the year prior. This association was mostly explained by return visits as new patient visit adherence was not impacted by the pandemic. Initiatives should be undertaken to determine the effects of pandemic-induced appointment nonadherence.

\section{EPV166/\#226 GYNECOLOGICAL CANCER SURGERIES IN TERTIARY CARE HOSPITAL OF PAKISTAN IN THE ERA OF COVID-19 PANDEMIC}

F Dahar*, U Chishti, A Begum. Aga Khan University Hospital, Obstetrics and Gynaecology, Karachi, Pakistan

\subsection{6/ijgc-2021-IGCS.236}

Objectives COVID-19 pandemic has affected the systems in all hospitals and non-essential elective surgeries were deferred. In this retrospective study we have evaluated results and complications of gynaecological cancer surgeries in a tertiary care hospital during the first 9 months of covid pandemic in our country.

Methods We retrospectively analyzed the medical charts of patients who underwent these surgeries from March-December, 2020.

Results The study included 116 patients, 48 endometrial, 50 ovarian, 14 cervical and 4 vulval \&vaginal cancers. Majority of cancers were early stage (64\%). The median age was 58 years (range 22-85years). Surgical approach was laparotomy in $77.6 \%$ including $48 \%$ complex surgeries. Based on the BGCS framework for prioritization of these surgeries, most of our surgeries belong to priority level 2(89\%) and 3(11\%). COVID verbal screening (by a questionnaire) was done in $90 \%$ of patients starting in Mid-March. Formal COVID testing by PCR for all pre-operative patients was commenced in April and hence $89(77 \%)$ of all patients underwent this testing. Only 2 patients were found COVID positive and the surgery was deferred for 4 weeks. Complications based on ClavienDindo grade 1 , grade $4 \mathrm{a}$ and grade 5 were observed in 4 patients. Median hospital stay was 5 days. Out of 12 patients with clinical suspicion of COVID within 30 days of surgery 3 were found to be covid positive, including one requiring ICU admission. 